\title{
Dental abscess: A potential cause of death and morbidity
}

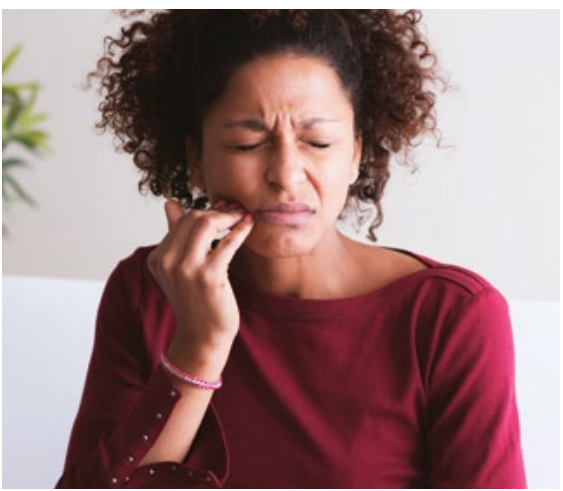

CPD

Kristen Bayetto, Andrew Cheng

Alastair Goss

\section{Background}

Dental abscess as an end stage of dental disease is common in the community, and patients with dental abscesses are likely to seek care from their primary health provider. Once the infection has spread beyond the confines of the jaws, there is an increasing risk of airway obstruction and septicaemia. If treated with antibiotics alone, the infection will not resolve and will become progressively worse.

\section{Objective}

This article reviews the pathophysiology, demographics and management of severe odontogenic infections. It includes evidence-based studies of a large number of cases treated at a single tertiary hospital.

\section{Discussion}

Prompt assessment and referral to a tertiary hospital is required for cases at risk of airway compromise. The morbidity and mortality of cases is presented in this article, with discussion of risk factors and the financial burden on the health system.
PATIENTS WITH DENTAL ABSCESSES commonly initially present to their primary health providers, particularly if they have dental phobia or are financially constrained. It is easy to underestimate their condition, particularly if the infection has spread beyond the confines of the jaws. In the pre-antibiotic era, dental infection was a common cause of death, with fatality rates of $10-40 \% .{ }^{1}$ With the advent of antibiotics, odontogenic infections responded well to penicillin. This may have created a false sense of security, and such cases were usually treated by junior hospital staff operating out of hours. It may not have been noticed that antibioticresistant bacteria were generally on the increase. This was the situation at the Royal Adelaide Hospital in 2002 when a patient with a spreading odontogenic infection died of airway obstruction a few hours after operation (Case 1). This event resulted in an immediate review of the management of such cases, and an audit of all 88 inpatient cases treated in the year 2003 was performed. ${ }^{2}$ These key steps were completed by the time of the coronial investigation in $2006 .^{3}$ Subsequently, there has been a further detailed audit of 672 patients in the period 2006-14. ${ }^{4}$ Altogether, more than 1000 cases of severe odontogenic infection have been managed at the Royal Adelaide Hospital in the period 2002-19. This evidence-based experience forms the basis of this article.

In this article, the authors examine multiple factors associated with severe odontogenic infections, including:

- the evidence base for the pathophysiology, which includes the anatomical basis, microbiology and host factors

- patient demographics

- initial assessment of airway risk and assignment to low- or high-risk cases

- hospital management and outcome, morbidity and mortality

- identified risk factors

- the financial burden.

\section{Pathophysiology}

The onset of a dental abscess is usually slow over many months. Dental decay takes several months to reach the dental pulp. Pulpitis results in pain that is poorly localised. When pulp necrosis finally occurs, there is no pain. However, when an acute periapical abscess develops, a severe well-localised pain develops. At this stage, the dental abscess is easily treated by extraction or root filling. By this time, all patients have had intermittent episodes of pain as a warning that something is wrong. Other causes of dental abscess are pericoronal infections around partially 
erupted impacted teeth or failed dental treatment. ${ }^{2}$ Thus, there are clear warning symptoms; some patients ignore the symptoms while others receive temporary relief with antibiotics from medical or dental practitioners. Antibiotic treatment without dental treatment to remove the cause always fails. $^{2}$

Once the infection spreads beyond the confines of the jaws and into the soft tissue spaces, it becomes much more difficult to treat and potentially life threatening.

\section{Anatomical factors}

Anatomical factors play a key part in the progression of infection once beyond the confines of the teeth and jaws. Spread follows the line of least resistance, which is dictated by the fascia and muscles. ${ }^{5}$ The anatomical space involved depends on the affected tooth. The most dangerous space is the submandibular space, which is bounded by the mandible laterally, the mylohyoid muscle above and the subcutaneous tissue and skin below. It contains the submandibular gland, lymph nodes and the masseter muscle. When this muscle is irritated by the inflammation, trismus or difficulty in jaw opening ensues. The submandibular space is in direct contact with the pharyngeal spaces and down through the neck to the mediastinum. It also connects to the contralateral side. ${ }^{5}$

It is essential to understand that swelling from the submandibular spaces can extend over a wide area of the airway in both length and breadth (Figure 1).

This is the basis of the term 'Ludwig's angina', with angina meaning 'choking'. Strictly, Ludwig's angina only refers to cases in which the whole neck bilaterally from the mandible to the clavicle is involved in infection. Such cases have a poor prognosis. ${ }^{6,7}$ However, the term 'Ludwig's angina' is commonly misinterpreted to apply to any localised neck infection. ${ }^{3}$

The other critical area of dental infection is the incisors, canines and premolars in the anterior maxilla, as infections in these areas can spread via the infraorbital veins to the ocular veins to the cavernous sinus. Spread is facilitated as these veins have no valves.

\section{Microbiological factors}

Odontogenic infections are polymicrobial with a mixture of aerobic, facultative anaerobic and strict anaerobic organisms. The deeper the infection, the more likely that the involved organisms are anaerobic. ${ }^{4}$ Generally, the more skilled and intensive the microbiological study, the greater the range and type of bacteria will be shown. ${ }^{8}$ Current hospital bacteriological studies are usually brief, limited to indicating the general site (eg oral or respiratory). It is best to involve infectious disease consultants when complex resistant cases are encountered. The most common organisms are viridans streptococci initially, with the subsequent anaerobes being Fusobacterium spp. and Prevotella spp.

A small but clinically important subgroup of odontogenic infections is those with necrotising fasciitis. Clinically, these infections have extensive tissue destruction, with gas within the tissues and extensive spread. They are usually associated with Serratia spp., Klebsiella oxytoca, Enterococcus faecalis and Candida spp. Management of these cases involves infectious disease input, and patients face a long hospital stay. ${ }^{7}$

\section{Pre-existing medical conditions}

If the patient is immunocompromised (eg with human immunodeficiency virus/acquired immunodeficiency syndrome, haematological neoplasms or poorly controlled diabetes), there is likely to be increased difficulty in management and a longer hospital stay for the patient.
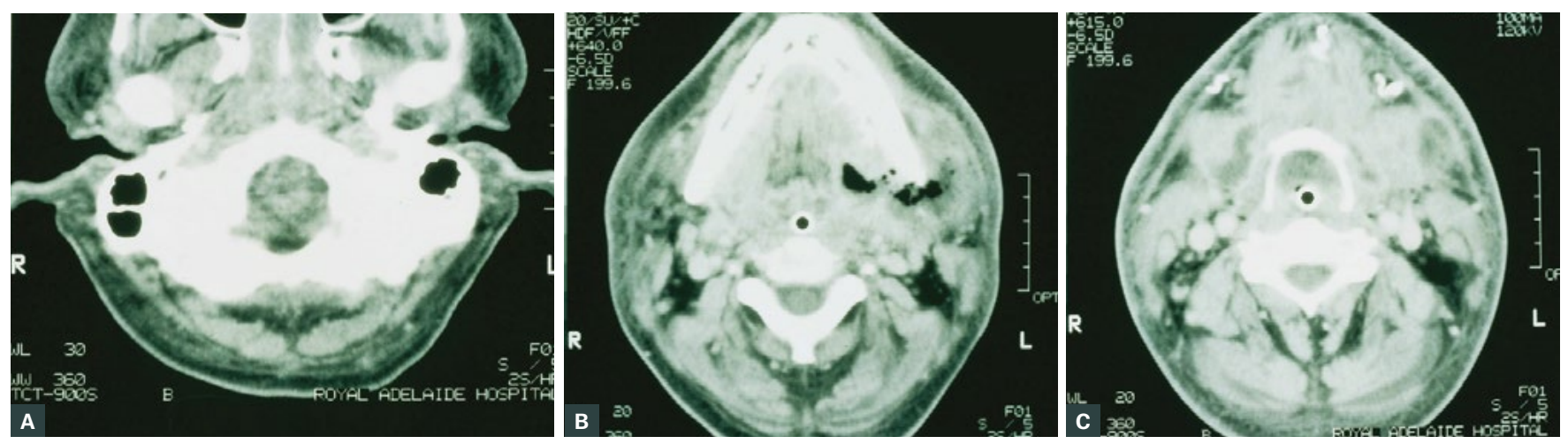

Figure 1. Serial axial computed tomography slices showing the extent of airway compromise in a patient with a left submandibular abscess who was orally intubated

A. Complete obstruction of the nose and nasopharynx; B. Left aubmandibular pus collection and oropharyngeal obstruction around the tube;

C. Hypopharyngeal obstruction at the level of the hyoid bone with a shift of the airway to the right

Reproduced with permission from Uluibau I, Jaunay, T, Goss A, Severe odontogenic infections, Aust Dent J 2005;50(4 Suppl 2):S74-S81, doi: 10.1111/j.18347819.2005.tb00390.x. 
Older patients with cardiovascular and respiratory disease have increased difficulty coping with the rigors of surgery and intensive care management.

\section{Preliminary assessment}

The key first step is to assess whether the patient is low or high risk for airway embarrassment (Box 1). This begins with a thorough history and clinical examination. It is important to determine whether this is the first episode or infection or whether the patient has had previous courses of antibiotics. It may also be beneficial to enquire why they have not seen a dentist. Infections below the lower border of the mandible, including the deep neck spaces, have an increased airway risk. A clinical indicator of airway compromise is trismus; therefore, it is important to determine

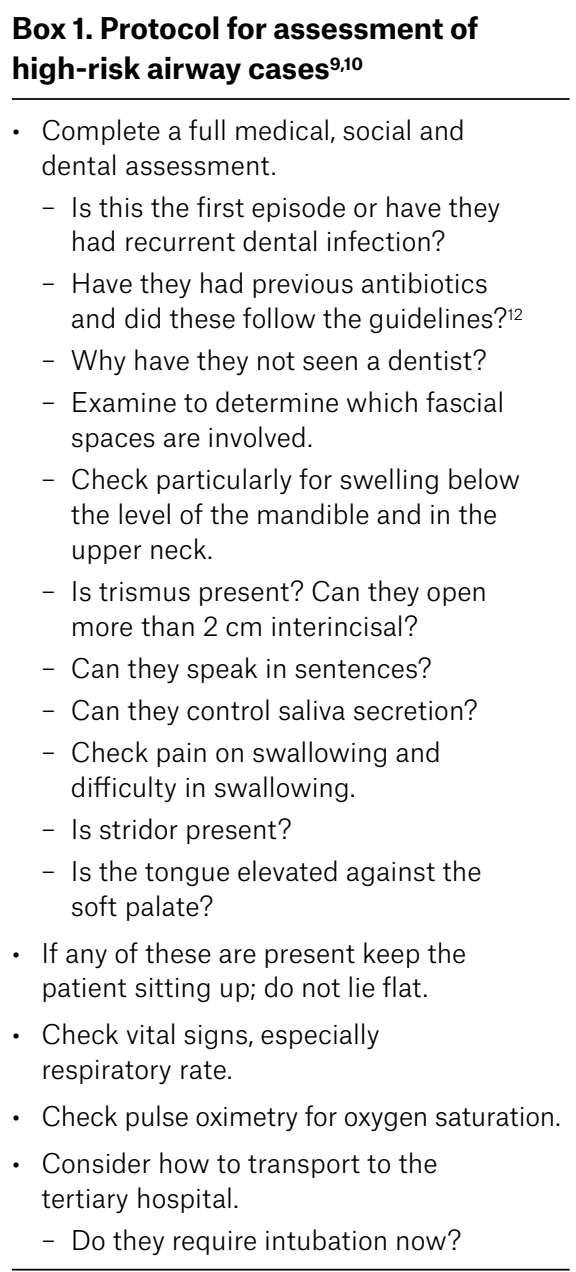

whether the patient's mouth opens $<2 \mathrm{~cm}$ between the teeth.

Questions should also be asked relating to swallowing - whether they have pain on swallowing and difficulty swallowing, and whether they can speak in sentences and swallow their own saliva. If the patient has stridor on inspiration and their tongue raised against the soft palate, they must be kept sitting upright and not laying down, as that will increase their risk of obstruction.

Patients with a high risk of airway obstruction should be promptly transferred to a tertiary hospital. If they are being transferred any distance by road or air, intubation should be considered. Patients without these signs of airway compromise are low risk.

Patients with decay and pulpitis, or early abscess, should be referred to a general dentist. This should follow the usual referral process; that is, a letter to a specific dentist, either the patient's choice or one known to the primary healthcare provider. It is insufficient just to tell the patient to go to a dentist.

\section{Royal Adelaide Hospital cohort Demographics}

Patients with spreading odontogenic infections covered a wide demographic, with an average age in the thirties but a range from 18 to 95 years. ${ }^{2,4}$ Patients under the age of 18 years were treated separately at the Adelaide Women's and Children's Hospital. There was a small predominance of men over women: $55 \%$ to $45 \%$.

The most common medical issues were mental illness and substance abuse. This socially disadvantaged group has increased dental decay secondary to drug-related hyposalivation and commonly does not access public dental care. Aboriginal and Torres Strait Islander patients were also overrepresented.

\section{Tertiary hospital assessment}

In South Australia, all spreading odontogenic infections are transferred to the Royal Adelaide Hospital, where there is a standardised multidisciplinary protocol with input from oral and maxillofacial surgery; ear, nose and throat surgeons; anaesthetists; the intensive care unit (ICU) and the infectious disease unit. ${ }^{9}$ Sixty-four per cent of patients were assessed as being at high risk of airway obstruction, while $36 \%$ were determined to be at low risk, with only superficial infections.

The airway was assessed and, if needed, promptly intubated. If antibiotics had not already been commenced, patients were given high-dose intravenous penicillin and metronidazole. Imaging of the jaw and teeth was performed with orthopantomography. Computed tomography (CT) scans were not routinely performed; if they were, it was only after the airway had been secured. Patients proceeded to theatre within a few hours for removal of the causative teeth and incision and drainage of the involved spaces. Drains were left in situ. Blood tests and microbiological swabs were obtained. Progress was then monitored, with antibiotics changed on the basis of antibiotic resistance results. If patients did not respond, then a CT scan was performed to determine if there were other pus collections, in which case patients were returned to theatre for further drainage. The patient was only extubated when they could clearly breathe around the tube. The decision to extubate was made by the consultants responsible following laryngoscopic examination and not by junior staff at night. The average length of time spent in the ICU was $38.5 \pm 60.4$ hours. The patient was then transferred to the ward for further oral antibiotics, removal of the drains when there was no remaining suppuration, and monitoring.

\section{Outcomes}

Most patients recovered fully and were discharged within a week, with low-risk patients remaining in hospital for $1.9 \pm 1.3$ days and high-risk patients staying for $5.1 \pm 4.9$ days. Persistent firm swelling in the region of the infection, scars from the incision and drainage, and persistent trismus were common. These sequelae took 1-2 months to settle. Psychological issues, particularly for patients with pre-existing mental issues, were common.

Some patients had longer inpatient stays with increased morbidity. Increased length of stay was more closely related to 
bilateral space involvement than unilateral space involvement (60\% increase). Each year of age added $1 \%$ to the length of stay. Length of stay was particularly increased for high-risk cases that involved ICU time; for these patients, length of stay was 59\% longer than for those who did not require prolonged intubation.

In a separate study of 256 patients admitted to an ICU between 2008 and 2013, 230 patients had odontogenic infections. Of these patients, all were intubated for an average of 1.5 days (range: 0.7-2.6 days). Forty-eight per cent had medical comorbidities, $2 \%$ required a tracheostomy and $1 \%$ had ventilatorassociated pneumonia. Two patients died in the ICU, one of ventilator-associated pneumonia and one of odontogenic septicaemia. ${ }^{10}$

Examples of survival with serious morbidity included descending necrotising mediastinitis (Figure 2), with another patient developing a cerebral mycotic aneurysm of the brain (Figure 3) with cavernous sinus thrombosis. Another developed facial necrosis with blindness and required extensive rehabilitation (Case 2).

Five patients died during the study period. One died of airway obstruction (Case 1). None have died of airway obstruction since the airway protocol was instituted in 2002. One patient died of prolonged ventilation respiratory pneumonia that was acquired while in

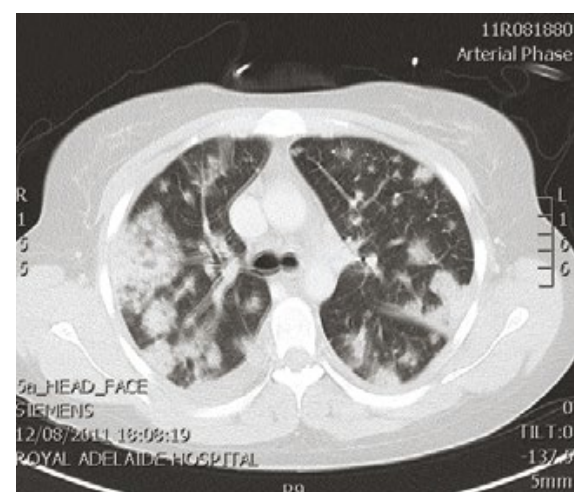

Figure 2. Mediastinal and lung involvement from a spreading odontogenic infection the ICU. She was elderly and medically compromised, and the odontogenic infection had been resolved. Three patients died of odontogenicrelated septicaemia. All had medical comorbidities and antibiotic resistance on presentation.

In an attempt to further characterise risk factors, a detailed study was performed on antibiotic resistance. The study found that $10.8 \%$ of patients had penicillinresistant organisms. All had multiple prior courses of antibiotics, either for the odontogenic infection or other conditions or both. As soon as antibiotic resistance was discovered, they were changed to broad-spectrum antibiotics on the advice of the infectious diseases service. It is recommended that antibiotic prescribing follows Therapeutic Guidelines, 'Oral and dental', Version 3. ${ }^{11}$

Finally, the authors assessed the financial burden of spreading odontogenic infections on the healthcare system. The burden is significant, with the average cost for high-risk patients being $\$ 12,228$. The total cost over the seven-year period was $\$ 5.65 \mathrm{M}$. This needs to be compared with the average cost of a single tooth extraction in private dental practice, which is $\$ 181$ (125 times less expensive). ${ }^{12}$

\section{Conclusion}

Dental abscess is a common preventable disease that can be simply treated in

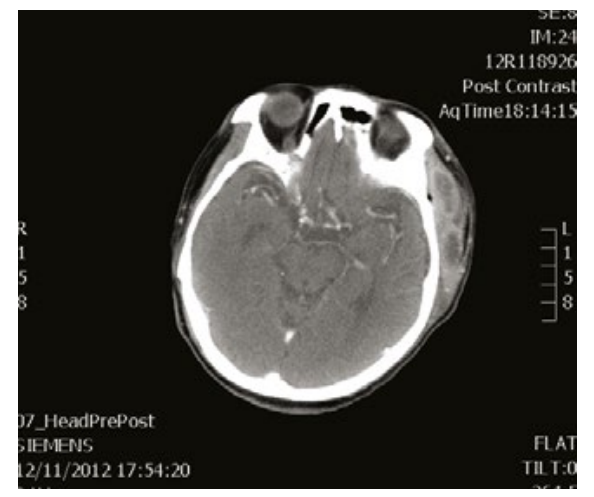

Figure 3. Cerebral mycotic aneurysm and cavernous sinus thrombosis from a spreading odontogenic infection general dental practice. Unfortunately, less than half of all Australians have regular or emergency-only dental care. ${ }^{13}$ If an abscess spreads beyond the tooth, it requires dental treatment and will not respond to antibiotics alone. If the infection spreads into the fascial planes of the neck or face, then there is risk of airway compromise or spread to the brain. Appropriate protocols of management have been established at tertiary hospitals, but there is still and morbidity and mortality.

The condition of dental abscess can be prevented, but this requires better access to dental care and careful antibiotic stewardship by all health professionals.

CASE 1

A man aged 27 years, who was otherwise medically fit but a heavy smoker, developed a toothache. He took no action beyond analgesics and at least one course of antibiotics over a four-month period. He then presented to a secondary hospital with a unilateral submandibular swelling and was directed to the Royal Adelaide Hospital. He presented there 12 hours later and was admitted.

Under general anaesthesia, he had an incision and drainage of the abscessed right submandibular space. A general dentist had evidently previously extracted the tooth. As the patient had trismus, he had a difficult fibreoptic intubation. At the end of the procedure he was extubated and sent to the ward.

He recovered from the anaesthetic, had a shower and rang his mother to tell her he was doing well. His mother felt that he was not able to talk properly and told the nurse. The nurse said she would call the surgeon who performed the procedure and told the patient to return to his bed. He lay down and promptly developed airway obstruction.

The surgeon and crash team were present in $<2$ minutes and attempted firstly to intubate him then to perform an emergency tracheostomy. These attempts failed, and the patient died. ${ }^{3}$

This case resulted in a major change in protocol for the management of patients with airway compromise. 


\section{CASE 2}

A woman aged 32 years who was medically well but used intravenous drugs presented to the Royal Adelaide Hospital with a two-week history of increasing facial swelling and trismus. In the two-week period she had obtained two courses of antibiotics from a locum medical service and was self-medicating for the pain with street drugs.

On admission she had gross oral sepsis, perioral necrotising fasciitis and multi-organ dysfunction. On the day of admission, she was intubated and had a full dental clearance, drainage of multiple infected spaces and debridement of necrotic tissue. Wound swabs grew both community-acquired methicillin-resistant Staphylococcus aureus and Candida tropicalis. The patient had little improvement despite multiple drainages, debridement of necrotic tissue and involvement of an infectious disease consultant. She developed cavernous sinus thrombosis and had a cerebrovascular accident with unilateral paresis and decreased vision. She spent many weeks in intensive care and was eventually discharged to the rehabilitation centre at 180 days. The patient survived but has multiple permanent impairments including hemiparesis, partial blindness and cognitive impairment. ${ }^{7}$
3. Coroner's Court of South Australia. Findings of Inquest - Daniel Brindley Salmon. Inquest number 27, 2006. Available at www.courts.sa.gov.au/ CoronersFindings/Lists/Coroners\%20Findings/ Attachments/341/SALMON\%20Daniel\%20 Brindley.pdf [Accessed 24 June 2020].

4. Liau I, Han J, Bayetto K, et al. Antibiotic resistance in severe odontogenic infections of the South Australian population: A 9-year retrospective audit. Aust Dent J 2018;63(2):187-92. doi: 10.1111/ adj.12607.

5. Grodinsky M, Holyoke EA. The fasciae and fascial spaces of the head, neck and adjacent regions. Am J Anat 1938;63:367-408.

6. Laskin DM. Anatomic considerations in diagnosis and treatment of odontogenic infections. J Am Dent Assoc 1964;69:308-16. doi: 10.14219/jada. archive.1964.0272.

7. Juang YC, Cheng DL, Wang LS, Liu CY, Duh RW, Chang CS. Ludwig's angina: An analysis of 14 cases. Scand J Infect Dis 1989;21(2):121-25. doi: 10.3109/00365548909039957.

8. Sakamoto $H$, Kato H, Sato T, Sasaki J. Semiquantitative bacteriology of closed odontogenic abscesses. Bull Tokyo Dent Coll 1998;39(2):100-07.

9. Central Northern Adelaide Health Network, Standards and Procedures Committee. Acute head and neck infections protocol. Adelaide SA: Royal Adelaide Hospital. Standards and Procedures Committee 2012.

10. Sundararajan K, Gopaldas JA, Somehsa H, Edwards S, Shaw D, Sambrook P. Morbidity and mortality in patients admitted with submandibular space infections to the intensive care unit. Anaesth Intensive Care 2015:43(3):420-22.

11. Oral and Dental Expert Group. Therapeutic Guidelines: Oral and dental. Version 3. Melbourne, Vic: Therapeutic Guidelines Limited, 2019.

12. Han J, Liau I, Bayetto K, et al. The financial burden of acute odontogenic infections: The South Australian experience. Aust Dent J 2020;65(1):39-45. doi: 10.1111/adj.12726.

13. Ju X, Brennan DS, Spencer AJ. Age, period and cohort analysis of patient dental visits in Australia. BMC Health Serv Res 2014;14:13. doi: 10.1186/1472-6963-14-13.

\section{Authors}

Kristen Bayetto MBBS, BDS, Advanced Oral and Maxillofacial Surgery trainee, Royal Adelaide Hospital, SA

Andrew Cheng MBBS, BDS, FRACDS (OMS), Consultant Oral and Maxillofacial Surgeon, Royal Adelaide Hospital, SA

Alastair Goss DDSc, FRACDS (OMS), Emeritus

Consultant, Royal Adelaide Hospital, SA; Emeritus Professor, Oral and Maxillofacial Surgery, The University of Adelaide, SA. alastair.goss@adelaide.edu.au Competing interests: None.

Funding: None.

Provenance and peer review: Commissioned, externally peer reviewed.

\section{References}

1. Wilwerding T. History of dentistry 2001. Available at www.freeinfosociety.com/media/pdf/4551.pdf [Accessed 24 June 2020].

2. Uluibau IC, Jaunay T, Goss AN. Severe odontogenic infections. Aust Dent J 2005;50(4 Suppl 2):S74-S81. doi: 10.1111/j.1834-7819.2005.tb00390.x. 